\title{
Disks and Jets in High-Mass Young Stellar Objects
}

\author{
Riccardo Cesaroni \\ Osservatorio Astrofisico di Arcetri, Firenze, Italy
}

\section{Introduction}

The formation of stars is a complex process which is poorly understood at present, although recently important progress has been made both on a theoretical and observational ground. It is clear that star formation must proceed through contraction of a large molecular clump into a dense optically thick protostellar core: the obvious consequence is that conservation of angular momentum must force the material to spin up and flatten. Thus, formation of disks around newly formed stars is a very sensible expectation. Indeed, the recent development of instruments like the Hubble Space Telescope (HST) and the millimeter interferometers has allowed detection of several disks around low-mass young stellar objects (YSOs), such as the Keplerian disk in GG Tau (Guilloteau et al. 1999) and that seen with the HST in HH 30 (Burrows et al. 1996). The situation is quite different for high-mass YSOs. In this case, the evidence for disks is scarce, although a priori one would expect these to be more massive than those in low-mass YSOs and hence easier to detect. Various effects may complicate this simple-minded picture: for instance, magnetic field is likely to play an important role coupling the inner part of the collapsing cloud to its outer layers, thus making angular momentum conservation difficult to apply to any single "portion" of the cloud; depending on the ratio between disk and stellar mass, the disk may be unstable and hence short-lived; the effects of the stellar wind and radiation have to be taken into account; finally, the mass and size of the disk depend on the accretion process, which is not well understood. All these caveats probably explain why disks around massive YSOs are difficult to detect.

It is also important to stress that the formation of the disk and the ejection of a jet/outflow from the newly formed star might be strictly related. Therefore, one should investigate both phenomena at the same time when studying the formation of stars. In the following, we will summarise the current evidence for disks in massive YSOs and the relevance of jets as a tool to prove their existence.

\section{Evidence for disks in massive YSOs}

Unlike the case of low-mass stars, a complete theory for the formation of massive stars has not been developed yet and it is hence difficult to guess the expected size of disks in these objects. However, it seems unlikely that they can be much greater than $1000 \mathrm{AU}$, which correspond to $1^{\prime \prime}$ at a distance of $1 \mathrm{kpc}$. Since the majority of massive stars are located at much larger distances, the disk angular diameters are expected to be $\lesssim 1^{\prime \prime}$, namely comparable to the angular resolution of the millimeter interferometers currently available. Furthermore, unlike low- 
mass stars, massive ones reach the zero age main sequence still deeply embedded in their parental cloud, with visual extinctions up to 1000 mag, which makes impossible to detect the disks even at NIR wavelengths. Maser lines are instead an excellent probe for these dense regions: in fact, they are emitted in the radio regime, where extinction is negligible, and trace the velocity field of the molecular gas on scales as small as a few AU, where they can be observed thanks to VLBI measurements at centimeter wavelengths. However, masers can sample the gas only in a limited number of points, whereas thermal lines - although observable with much worse angular resolution - have the advantage to sample the bulk of the molecular gas. It is hence clear that both thermal and maser lines are necessary to investigate the global structure of disks.

Table 1. Possible disks in high-mass YSOs

\begin{tabular}{|c|c|c|c|c|c|}
\hline Tracer & Source & $\begin{array}{c}M_{*} \\
\left(M_{\odot}\right)\end{array}$ & $\begin{array}{l}M_{\text {Disk }} \\
\left(M_{\odot}\right)\end{array}$ & $\begin{array}{l}R_{\text {Disk }} \\
(\mathrm{AU})\end{array}$ & Reference \\
\hline$\overline{\mathrm{CH}_{3} \mathrm{OH}}$ & $\begin{array}{l}\text { UC HII } \\
\text { regions }\end{array}$ & $\geq 0.5-73$ & $\overline{-}$ & $\geq 95-3700$ & $\begin{array}{l}\text { Norris et al. } 1998 \\
\text { Walsh et al. } 1998\end{array}$ \\
\hline $\mathrm{SiO}$ & Orion-KL & $\overline{4.4}$ & - & 80 & $\begin{array}{l}\text { Barvainis } 1984 \\
\text { Plambeck et al. } 1990\end{array}$ \\
\hline$\overline{\mathrm{H}_{2} \mathrm{O}}$ & $\begin{array}{l}\text { Ceph A } \\
\text { W75N(B) }\end{array}$ & $\begin{array}{l}20 \\
?\end{array}$ & $\begin{array}{l}70 \pm 40 \\
\leq 8\end{array}$ & $\begin{array}{l}300 \\
200\end{array}$ & $\begin{array}{l}\text { Torrelles et al. } 1996,1998 \\
\text { Torrelles et al. } 1997\end{array}$ \\
\hline $\begin{array}{l}\mathrm{C}^{18} \mathrm{O} \\
\mathrm{H}_{2} \mathrm{O} \\
\end{array}$ & G192.16-3.82 & $5-10$ & $7-32$ & 500 & $\begin{array}{l}\text { Shepherd et al. } 1998 \\
\text { Shepherd \& Kurtz } 1999\end{array}$ \\
\hline $\mathrm{CS}$ & G92.67+3.07 & $4-7$ & $10-20$ & 7000 & Bernard et al. 1999 \\
\hline $\begin{array}{l}\mathrm{CH}_{3} \mathrm{CN} \\
\mathrm{NH}_{3}\end{array}$ & $\begin{array}{l}\text { Hot Cores: } \\
\text { W3 OH, G10.62, } \\
\text { G10.47, G19.61, } \\
\text { G29.96, G31.41, } \\
\text { W51e2, W51e8, } \\
\text { IRAS } 20126\end{array}$ & $\begin{array}{l}\mathrm{O}-\overline{\mathrm{B}} \\
\text { stars }\end{array}$ & $10-10^{3}$ & $10^{3}-10^{4}$ & $\begin{array}{l}\text { Keto et al. } 1987 \\
\text { Wink et al. } 1994 \\
\text { Cesaroni et al. } 1994 \\
\text { Olmi et al. } 1996 \\
\text { Cesaroni et al. } 1997,1999 \\
\text { Zhang et al. } 1998 \mathrm{a}, 1998 \mathrm{~b}\end{array}$ \\
\hline
\end{tabular}

In Table 1 we summarise the current evidence for disks associated with highmass YSOs. With "high-mass" we will refer to (proto)stars with luminosities above $10^{3} L_{\odot}$. Such an evidence basically consists of the detection of flattened cores or chains of maser spots, with velocity gradients along their major axis. In the table we give the observed tracer, the source name, the mass of the star, the mass and radius of the disk, and the corresponding reference.

\section{Problems and conclusions}

Although several of the examples listed in Table 1 look quite convincing, still an iron-clad detection of a disk around a massive YSO has to be found. Various problems can hinder a fully convincing detection. Sometimes the "disk" is just an effect of poor angular resolution, and it "disappears" once better observations are available. In other cases, different tracers reveal velocity gradients in completely different directions. However, the problem which affects most of the cases is the lack of an independent check on the direction of the disk axis: under this respect, the detection of a jet perpendicular to the plane of the disk is a crucial 
test on the disk hypothesis. Only few of the sources in Table 1 are associated with a jet/outflow, coincident with the axis of the disk: the best examples are G192.16-3.82 and IRAS 20126+4104. In particular, the latter has been studied in great detail demonstrating that the jet does not bend going from a scale of $\sim 0.1 \mathrm{pc}$ (Cesaroni et al. 1999) to $\sim 100 \mathrm{AU}$ (Moscadelli et al. 2000) and is perpendicular to the Keplerian disk seen in the $\mathrm{NH}_{3}$ (Zhang et al. 1998b) and $\mathrm{CH}_{3} \mathrm{CN}$ lines on $\sim 2000 \mathrm{AU}$. The advent of new (sub) millimeter interferometers such as ALMA will dramatically improve the angular resolution of molecular line observations, thus making possible to confirm the existence of disks around massive YSOs by resolving their structure and deriving their rotation curve.

For the time being, we can only conclude that very likely disks exist also in high-mass YSOs, with masses of $10-10^{4} M_{\odot}$ and radii of $10^{2}-10^{4} \mathrm{AU}$. Despite these are several orders of magnitude larger than in low-mass YSOs, disks in massive YSOs remain elusive and this might be due to their shorter life-time, for which at least two explanations are possible: the fact that the disk is much more massive than the associated (proto)star, which makes it highly unstable; and the large luminosity, UV flux, and stellar wind of the O-B (proto)star, which accelerate the destruction of the disk.

\section{References}

Barvainis, R. 1984, ApJ, 279, 358

Bernard, J.P., Dobashi, K., Momose, M. 1999, A\&A, 350, 197

Burrows, C.J. et al. 1996, ApJ, 473, 437

Cesaroni, R. et al. 1994, ApJ, 435, L137

Cesaroni, R., Felli, M., Testi, L., Walmsley, C.M., Olmi, L. 1997, A\&A, 325, 725

Cesaroni, R. et al. 1999, A\&A, 345, 949

Guilloteau, S., Dutrey, A., Simon, M. 1999, A\&A, 348, 570

Keto, E.R., Ho, P.T.P., Haschick, A.D. 1987, ApJ, 318, 712

Moscadelli, L., Cesaroni, R., Rioja, M.J. 2000, A\&A, in press

Norris, R.P. et al. 1998, ApJ, 508, 275

Olmi, L., Cesaroni, R., Walmsley, C.M. 1996, A\&A, 307, 599

Plambeck, R.L., Wright, M.C.H., Carlstrom, J.E. 1990, ApJ, 348, 65

Shepherd, D.S., Watson, A.M., Sargent, A.I., Churchwell, E. 1998, ApJ, 507, 861

Shepherd, D.S., Kurtz, S.E. 1999, ApJ, 523, 690

Torrelles, J.M. et al. 1996, ApJ, 457, 107

Torrelles, J.M. et al. 1997, ApJ, 489, 744

Torrelles, J.M. et al. 1998, ApJ, 509, 262

Walsh, A.J., Burton, M.G., Hyland, A.R., Robinson, G. 1998, MNRAS, 301, 640

Wink, J.E. et al. 1994, A\&A, 281, 505

Zhang, Q., Ho, P.T.P., Ohashi, N. 1998a, ApJ, 494, 636

Zhang, Q., Hunter, T.R., Sridharan, T.K. 1998b, ApJ, 505, L151 